\title{
EXPERIMENTAL AND NUMERICAL ANALYSIS OF THE TENDON REPAIR PROCESS USING TUBULAR BRAIDED FABRICS
}

\author{
Jerry Ochola ${ }^{1 *}, B^{2}$ ny Malengier ${ }^{1}$, Lode Daelemans $^{1}$, John Githaiga ${ }^{2}$ and Lieva Van Langenhove ${ }^{1}$
}

1Department of Textiles, Ghent University, Technologiepark 907, Zwijnaarde 9052, Ghent, Belgium

${ }^{2}$ Moi University, Department of Industrial \& Textile Engineering, P.O. Box 3900 Eldoret, 30100 Kenya

*Corresponding author: Jerry.Ochola@UGent.be

\begin{abstract}
:
This paper presents the experimental and numerical analysis of the potential of a braided fabric for flexor tendon repair. Numerical models of tubular braided fabrics were generated using a python script interface and imported into ABAQUS $®$ while Flexor tendon models were represented as silicone rubber rods. Experimental tests and Finite Element Modelling (FEM) of the flexor tendon repair was undertaken by deploying two tendon ends from opposite sides of a tubular braided fabric. This was done such that the tendon ends meet at the midpoint within the fabric. The tendons were tightly held to emulate a realistic repaired tendon. A displacement driven uniaxial loading was induced on the tendon-fabric assembly sufficient to cause a $2 \mathrm{~mm}$ gap between the tendon ends. Numerical analysis of the repair potential of a braided fabric in tendon repair was done by analyzing selected fabric parameters that were crucial in tendon repair applications. The results show that changing the parameters of the braided fabrics significantly affected the potential of the fabrics during tendon repair.
\end{abstract}

\section{Keywords:}

fabric, braids, monofilament, finite element method, stress analysis

\section{Nomenclature}

\begin{tabular}{|c|c|c|}
\hline $\mathrm{D}_{\mathrm{T}}$ & $(\mathrm{mm})$ & Diameter of the tendon \\
\hline$D_{B}$ & $(\mathrm{~mm})$ & $\begin{array}{l}\text { Diameter of the braided fabric Yarn } \\
\text { diameter }\end{array}$ \\
\hline$\theta$ & $(\stackrel{\circ}{)}$ & Pitch angle between the braided yarns \\
\hline $\mathrm{L}_{\mathrm{B}}$ & $(\mathrm{mm})$ & Length of braided fabric \\
\hline CPRESS & (MPa) & ABAQUS $®$ Contact Pressure \\
\hline S, MISSES & (MPa) & ABAQUS $®$ Von Mises Stress \\
\hline$\Delta \theta$ & $(\stackrel{\circ}{)}$ & Angular reduction \\
\hline$\Delta \mathrm{R}$ & $(\mathrm{mm})$ & Radial contraction \\
\hline $\mathrm{D}$ & $(\mathrm{mm})$ & Displacement \\
\hline$\mu$ & $(-)$ & Coefficient of friction \\
\hline$\rho$ & $\begin{array}{c}(\mathrm{g} / \\
\left.\mathrm{cm}^{3}\right)\end{array}$ & Mass density \\
\hline
\end{tabular}

\section{Introduction}

Tubular braided fabrics are fabricated by helical interlacement of two sets of yarns in a circular manner; one set of yarns runs in the clockwise direction while the other runs in anticlockwise direction [1, 2].

Mechanical and geometrical modeling of braided fabrics have been the subject of diverse studies [3-9]. In the medical field, tubular braided fabrics have been adopted for numerous applications targeting mostly urology, the gastrointestinal tract, airway indications [10] and heart surgery as prosthetic stents
$[11,12]$. Traditionally, the repair of flexor tendons have been done using various suture techniques [13-14]. More recently, braided fabrics have been proposed for repair of ruptured tendons as possible replacement of the conventional methods of tendon repair. In this innovative approach [15], a braided fabric was adopted as a device for repair and rehabilitation of a tendon, as illustrated by Figure 1. In order to initiate healing and regeneration in ruptured tendons, it is necessary that a tendon repair technique offers adequate strength and ensures minimal gap formation at the repair site. Hence, analyzing the influence of fabric parameters on repair strength and gap formations is fundamental in the design of novel tubular braids capable of efficient tendon repair.

This paper therefore, provides insights into the performance of braided fabrics for tendon repair by undertaking an experimental and numerical analysis of the tendon repair process. Using finite element modeling, the performance of several braided fabrics were assessed for potential tendon repair applications. The force required to create a gap of 2 $\mathrm{mm}$ at the repair site was monitored, which was considered as a measure of the tubular braid's potential for a realistic tendon repair process. The finite element models considered in this paper provided realistic means to analyze this force and link it to the tubular braid deformation, pressure at the tendon-fabric interface and stress levels in the tendons. Furthermore, a parametric study was performed to determine the effect of yarn stiffness, tendon/yarn friction, initial pitch angle and yarn cross-sectional shape on the repair potential of braided fabrics. A series of experiments were performed to validate the FEM models. 


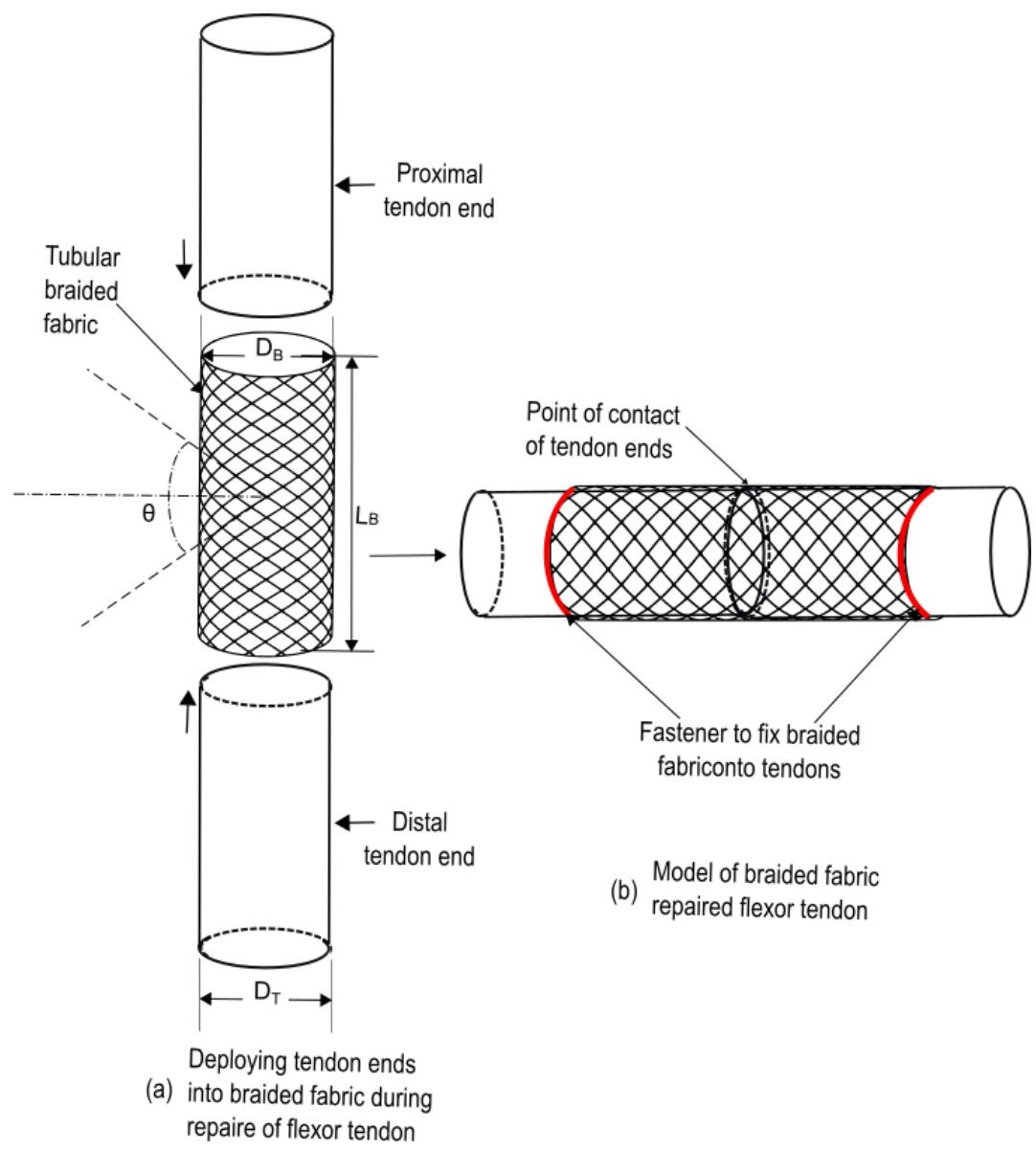

Figure 1: Schematic illustration of tubular braided fabric model and direction of deployment for tendon ends during tendon repair

\section{Materials and Methods}

\subsection{Geometrical model and material properties}

pyFormex (version 0.9.1) was used as a pre-processor to build the geometrical and finite element model of a tubular braided fabric in this study. The open source pyFormex software, currently under development at Ghent University, provides a Python based script to build a model by means of subsequent mathematical transformations. The investigated original braided fabric $\left(L_{B}=20 \mathrm{~mm}, D_{B}=8 \mathrm{~mm}\right)$ consists of single (regular-braided fabric) and double (multi-braided fabric) interlacing sets of helical yarns $\left(\theta=90^{\circ}\right.$ and yarn diameter $=$ $0.2 \mathrm{~mm}$ ) made from polyester (PET) monofilament yarns ( $E$ $=3500 \mathrm{MPa}, \mathrm{v}=0.3, \rho=1.3 \mathrm{gcm}^{-3}$ ) (see Figure 2). Samples with similar dimensions shown in Figure 2 were developed as geometrical models and also fabricated for experimental tests. The braided fabrics were meshed using linear elastic beam (B31) elements. The tendon material was represented using silicone rubber cords $\left(\rho=1.2 \mathrm{~g} \mathrm{~cm}^{-3}, \mathrm{v}=0.5\right)$; and modeled as three dimensional homogenous solids meshed with hexahedral (C3D8R) elements. The hyperelastic material properties of the rubber material was evaluated using $A B A Q U S \otimes$ Ogden material model [16] using actual test data sets from the silicone rubber samples considered in this paper. In this procedure, the constants for Ogden's Hyper-elastic model were automatically fitted by $A B A Q U S \circledast$ in terms material constants $\left(\mu_{1}, \alpha_{1}, D_{1}\right)$ using a non-linear least squares optimization procedure. The actual values for the parameters (evaluated from the test data) were: $\mu_{1}=21.1, \alpha_{1}=4.3$ and $D_{1}=0.0$, at $\mathrm{N}=1$.

\subsection{Experimental}

Following the methodology proposed by the innovations of Matthew Bills, [15], a model of flexor tendon repair using a tubular braided fabric was described as follows: (i) a rupture in the tendon was created by precisely cutting the artificial tendon prototypes at their mid region; (ii) the cut tendons were inserted into the tubular braid from opposite sides until they were just touching at the midpoint of the braided fabric; (iii) the tendon ends inserted in the fabric were fixed in place by fastening them onto the ends of the braid to minimize slippage. The

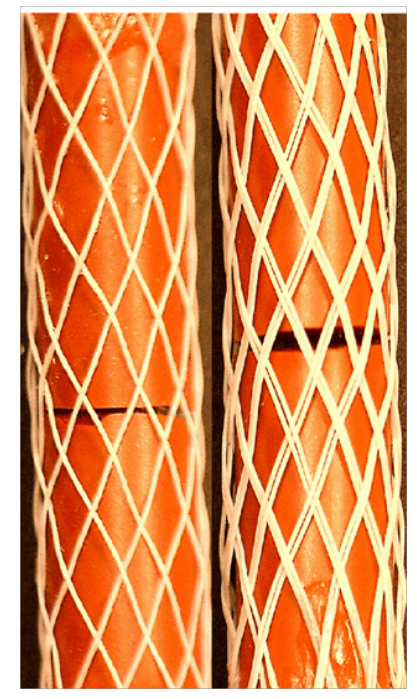

Figure 2: Samples of tubular braided fabrics and prototype tendon materials considered for experimental tests 
potential of the braided fabric in tendon repair was obtained by displacing one tendon end while fixing the opposite end. The samples were mounted in specially designed clamps of an Instron $®$ tensile testing machine and displaced at a rate of $5 \mathrm{~mm} / \mathrm{min}$. Since the goal of the study was to demonstrate the validity of the models in predicting the tendon repair potential of a tubular braided fabric, the repair models were run up to a gap size of $2 \mathrm{~mm}$ which is considered an allowable gap between tendons to allow healing and regeneration of the tissue [17-20].

The setup is as shown by the illustration in Figure 3. The gap formation was monitored during testing using a travelling digital microscope (low magnification). The test was stopped after a gap formation of $2 \mathrm{~mm}$ was obtained. The experimental data was used to validate the corresponding FE models.

\subsection{Meshing and Boundary Conditions}

The finite element mesh of the considered braided fabric was created automatically by pyFormex and subsequently imported into $A B A Q U S \AA$. The mesh for both braided fabric and tendon structure were selected after a sensitivity analysis in which the braided fabric was defined by 10240 (20480 for multi-braid fabric) two-node linear beam elements (B31); while the tendon structure was defined by 34000 hexagonal elements (C3D8R) (Figure 4).

The boundary conditions were defined by applying a displacement driven uniaxial loading on the distal tendon end, while the proximal tendon end is constrained in both the

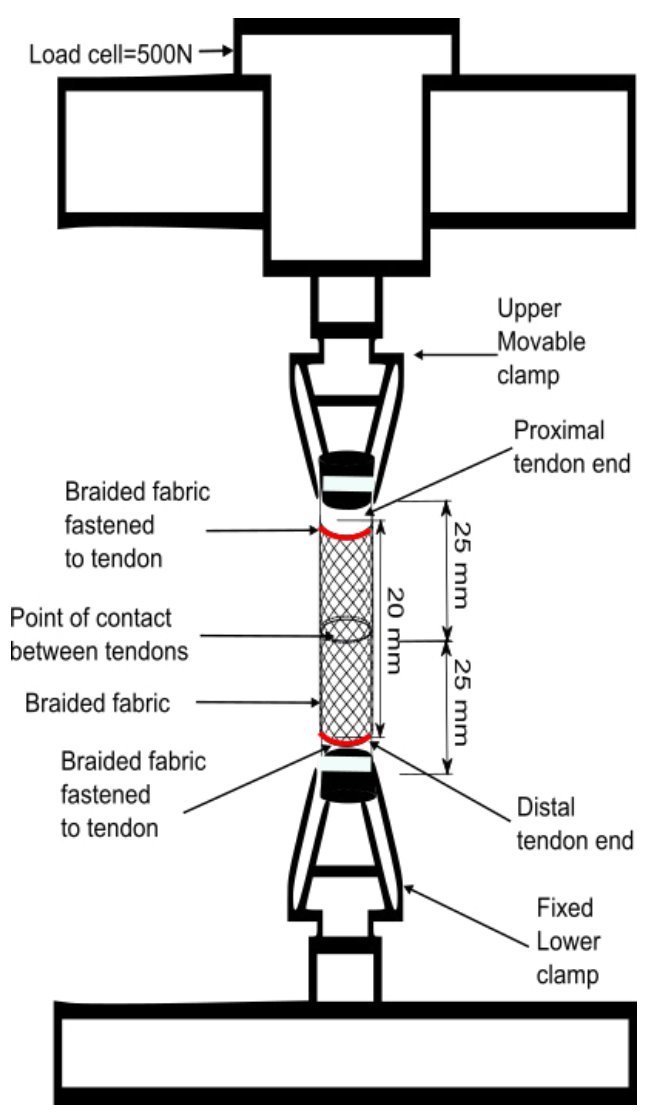

Figure 3. Experimental set up for uniaxial loading of the braided fabric repaired tendon axial and circumferential direction. These boundary conditions were able to emulate the actual tendon displacement behavior necessary for gap formation. Contact and friction at the yarnyarn interface and the tendon-yarn interface were applied using general contact algorithm with appropriate coefficient of friction as quoted from literature for similar materials under consideration. [21, 22]. The explicit solver ABAQUS $\otimes / E x p l i c i t$ was used in the simulations and the simulation time adjusted to have a quasi-static response, that is, kinetic energy is below $5 \%$ of the internal energy [23].

\section{Simulation Results and Experiment Verification}

The results of uniaxial loading of the tendons shown in Figure 5 and Figure 6, illustrate that the FEM results and the experimental test data for the study of the potential of braided fabrics in tendon repair are in reasonable agreement. In this study, the evolution of force on the tendons with displacement was considered up to a maximum gap of approximately $2.0 \mathrm{~mm}$ using a regular braided fabric (Figure 5 ) and a multi-braided fabric (Figure 6). It was observed that for the regular-braided fabric repair model, force increases as the displacement of tendons increase up to a maximum displacement of $12.0 \mathrm{~mm}$ when a gap of $2.0 \mathrm{~mm}$ was formed between the tendons; up to a maximum force of $17.6 \mathrm{~N}$ in the experiment and $17.1 \mathrm{~N}$ for the simulation.

In the multi-braided fabric repair model, the force on the tendons increased with displacement of the tendons. The tendon in the repair model was displaced by $20.0 \mathrm{~mm}$ for a 2.0 $\mathrm{mm}$ gap to be formed, of which the maximum force of $26.4 \mathrm{~N}$ was recorded for test data and $29.8 \mathrm{~N}$ for FEM data. In both the regular and multi-braid repair models (Figure 5 and Figure 6), the experimentally obtained force data was slightly higher than that predicted by the FE-models. This might be attributed to material non-linearity for both tendon and braided fabrics which are not accurately captured in the numerical computations. The results show that the multi-braided fabric repair model required

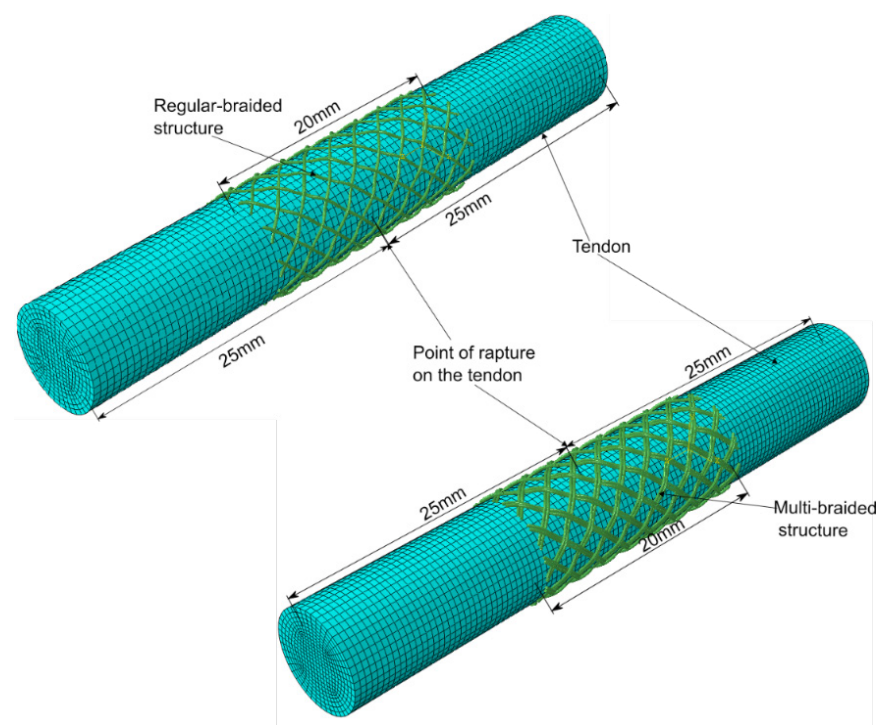

Figure 4: Geometrical models of the tendon-braid assembly for simulation of tendon repair using (i) regular-braided and (ii) multibraided fabrics. 
more force to form a gap of $2.0 \mathrm{~mm}(26.4 \mathrm{~N}$ for test data and $29.8 \mathrm{~N}$ for FEM) as compared to the regular braided fabric (17.6 $\mathrm{N}$ for test data and $17.1 \mathrm{~N}$ for FEM). This is linked to the fact that a multi-braided fabric would offer more resistance to gap formation. The gap resistance was established to be less than $30.0 \mathrm{~N}$ as reported by earlier research for gap resistance for some conventional surgical techniques of tendon repair [17-20]

Meanwhile, the results in Figure 5 and Figure 6 show slight difference between the experimental and numerical data. This is attributed to the fact that the FE models could not capture all the non-linearities in the actual test samples and can also be associated with the profile of ABAQUS $®$ amplitude applied in the uniaxial tensile loading protocol considered for the FE models. However, despite some differences existing between FE results and experimental data, the FE model developed in this paper could effectively be used to simulate the potential of a braided fabric in repair of tendons.

\subsection{Parameter study of tendon repair with reqular-braided fabrics using FEM}

The FE-models developed in this study were thus considered for an in-depth analysis and a parametric study was performed in order to assess the effect filament-material (elastic modulus) properties, contact-friction, initial pitch angles and yarn crosssectional shape on repair potential of the braided fabric.

The material stiffness of the braided yarns were changed from polyester monofilaments to nitinol wires. The material properties of nitinol was adopted from published literature [24]. The results were presented as shown in Figure 7 for the analysis of gap formation with force, contact pressure on tendons and

(i)

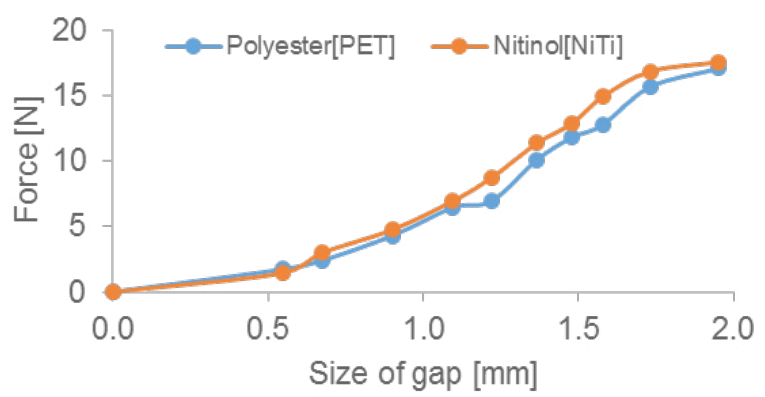

(iii)

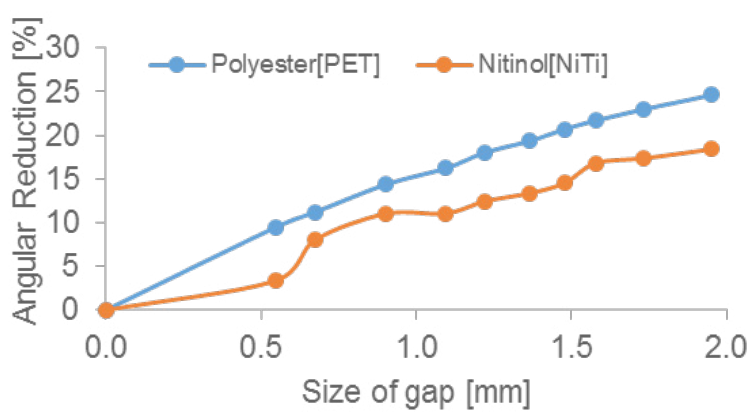

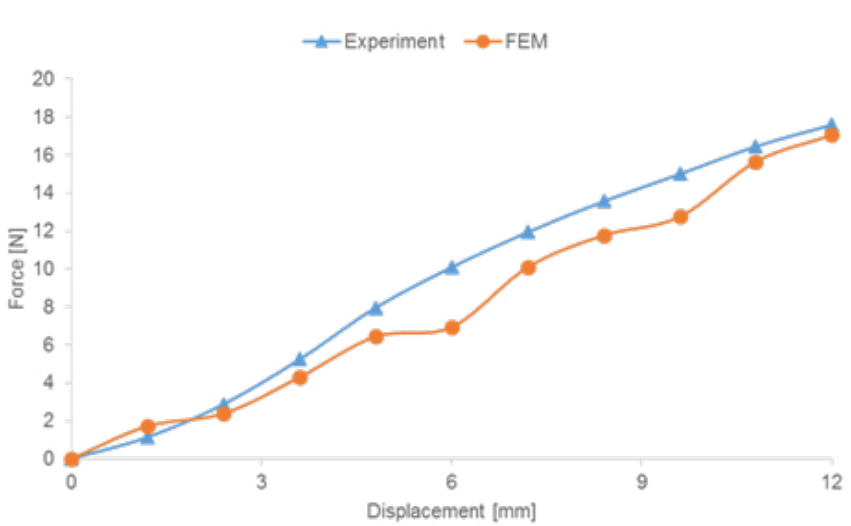

Figure 5: Experimental and FEM results for polyester-braided fabric repair models showing force-displacement analysis for regular-braided fabric model.

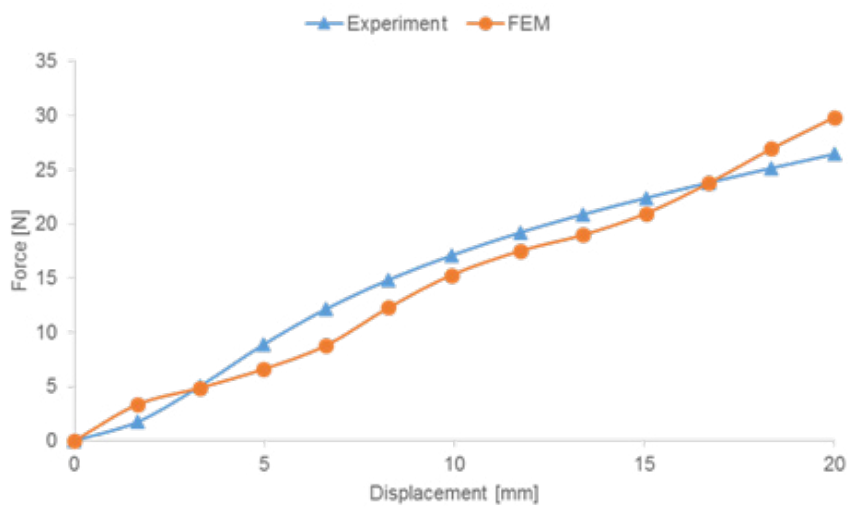

Figure 6: Experimental and FEM results for polyester-braided fabric repair models showing force-displacement analysis for multi-braided fabric model.

(ii)

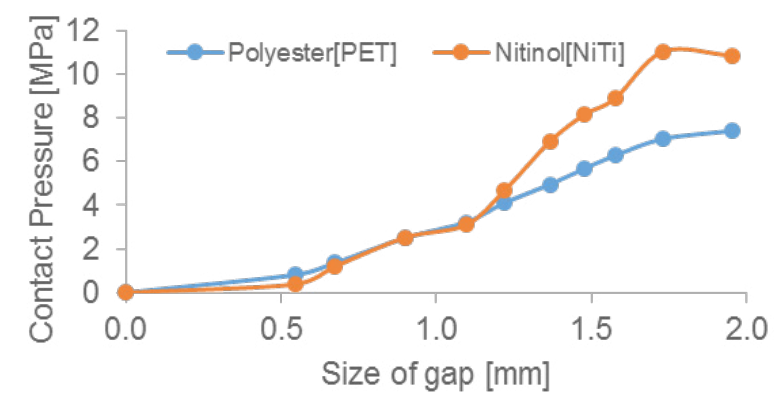

(iv)

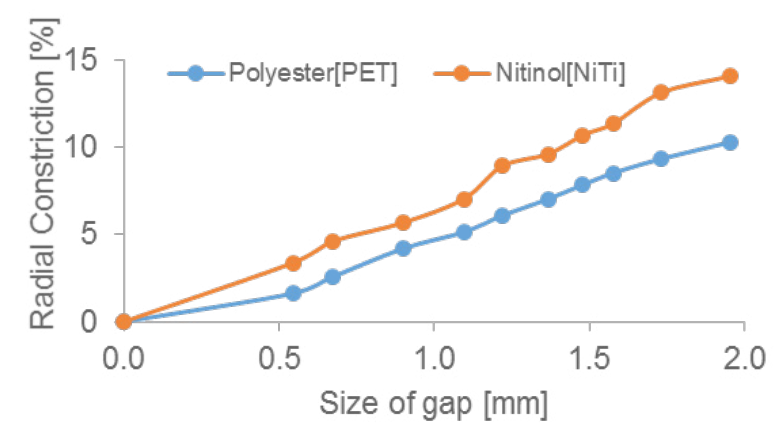

Figure 7: Analysis results for the braided fabric repair models showing (i) force - gap size, (ii) contact pressure - gap, (iii) percentage angular reduction - gap and (iv) percentage radial constriction - gap. 
deformation (angular reduction and radial constriction) for polyester and nitinol braided fabric repair models. The maximum displacement necessary for a $2.0 \mathrm{~mm}$ gap to be formed in both the polyester repair model and nitinol repair model was 12.0 $\mathrm{mm}$. At the maximum gap $(2 \mathrm{~mm})$, the results portray that the polyester braided fabric repair model had a maximum force of $17.1 \mathrm{~N}, 24.6 \%$ angular reduction, $10.3 \%$ radial constriction and $7.4 \mathrm{MPa}$ contact pressure at the tendon braid interface. Whereas in the nitinol braided fabric repair model at the maximum gap of $2.0 \mathrm{~mm}$, a maximum force of $17.5 \mathrm{~N}, 18.4 \%$ angular reduction and $14.1 \%$ radial constriction had a resultant contact pressure of $10.9 \mathrm{MPa}$ at the braid tendon interface. It was also noted from Figure 7 (i) and (ii) that the nitinol repair model had a higher force and contact pressure than the polyester repair model for most part of the gap formation. These results imply that the use of the nitinol braids would require more force to form the gap between the tendons than the polyester braids. Furthermore, as evidenced by the stress contours in Figure 8 and Figure 9, the contact pressure at the tendon braided interface was higher for the nitinol fabric model than for the polyester model. The results show relatively high stresses in the nitinol models which can be attributed to the higher stiffness of the nitinol material in the braided fabrics [21, 24].

There was also evidence of deformation in the braided fabrics due to uniaxial loading caused by the tendon displacement [2528], which was comparatively higher in the nitinol fabric repair model than in the polyester fabric repair model. This could be due to the fact that during gap formation, the tubular braid is stretched that causes radial contraction and angular reduction. In turn, this braid deformation causes a contact pressure and keeps both ends together resulting in a reaction force opposing the gap formation. The results in Figure 7 show that the contact pressure on the tendons on the repair model for both types of fabrics increase with an increase in the gap size which was consistent with the results of the evolution of force with gap size in both types of repair models. This could be attributed to the fact that as the tension in the tendons increased as evidenced by the increase in the force with gap size, the interface between the tendons and the braided fabric wrapped around them decreased.

This resulted into the tendon and the braided fabric tightening resulting into propagation of the contact pressure on the tendon surface which is evidenced by the contour plots in Figure 8(i) and Figure 9(i) than that in the nitinol repair model, even though the nitinol repair model braided fabric had more radial constriction than the polyester braided fabric. However, both fabrics indicated stress levels that are consistent with the stress levels of real tendons [29-31], indicating that the contact pressure due to the braided fabric could be within the limits of tendon stresses.

\subsubsection{Parameter analysis of repair potential of polyester braided fabric}

In the parameter study for the potential of the polyester braided fabric, it was noted that increasing the fabric contact friction,

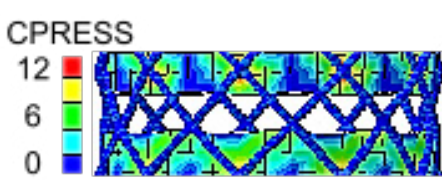

CPRESS

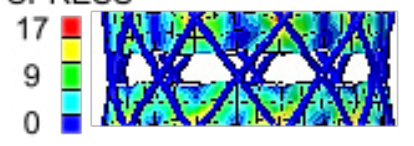

S, MISES

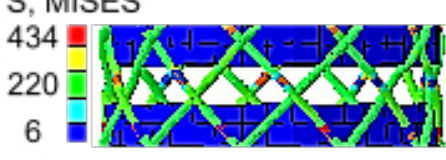

S, MISES

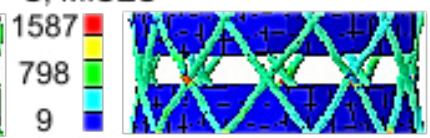

CPRESS

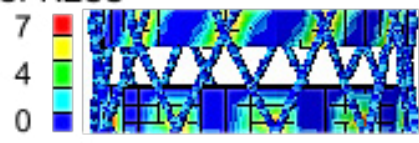

\section{S, MISES}

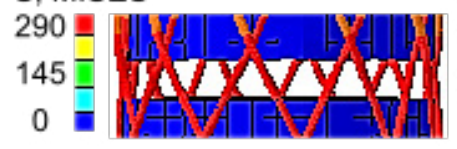

CPRESS

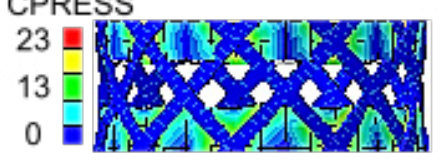

(i)

(ii)

$S$, MISES

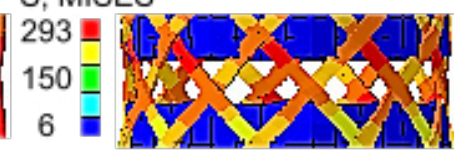

(iii)

(iv)

Figure 8: Contour plots for polyester braided fabric models showing contact pressure (top row) and Von Mises stress (bottom row) in tendonfabric interface (i) regular braided fabric repair model (ii) high friction repair model (iii) low initial pitch angle repair model (iv) tape yarn braided fabric repair model
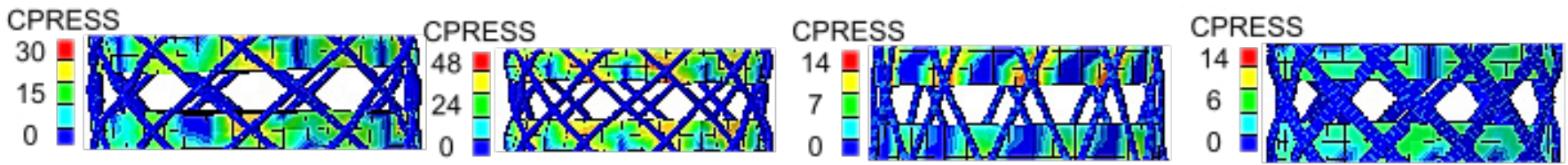

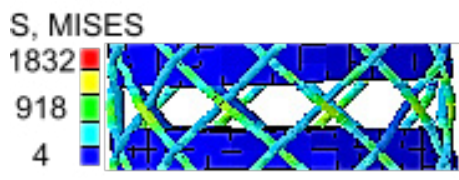

(i)

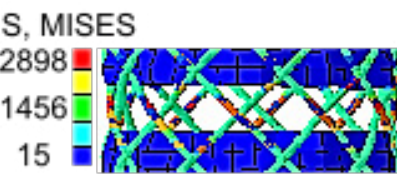

(ii)

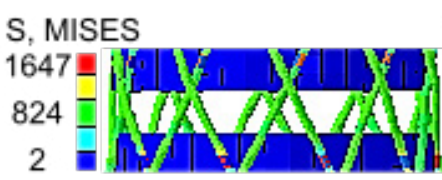

(iii)

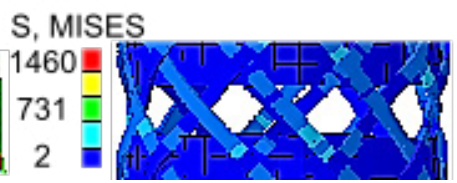

(iv)

Figure 9: Contour plots for nitinol braided fabric models showing contact pressure (top row) and Von Mises stress (bottom row) in tendon- fabric interface (i) regular braided fabric repair model (ii) high friction repair model (iii) low initial pitch angle repair model (iv) tape yarn braided fabric repair model 
the displacement of the tendons will be $22.0 \mathrm{~mm}$; while when a fabric with low initial pitch angle was reduced, the tendons were displaced by $12.0 \mathrm{~mm}$, same as when a tape yarn braided fabric was used. The results for the evolution of the gap between the tendons for different parameters analyzed are as shown by the plots in Figure 10.

In the analysis results summarized in Table 1 , it can be observed that when the parameters of the standard polyester braided fabric were changed, it was noted that in terms of force in the repair model required to form a $2.0 \mathrm{~mm}$ gap, changing the contact friction in the fabric will increase the force most $(35.6 \mathrm{~N})$, followed by using a fabric with a low initial pitch angle $(20.7 \mathrm{~N})$ and then using a tape yarn braided fabric $(17.6 \mathrm{~N})$. In terms of the contact pressure at the tendon-braid interface, a change in fabric friction had the most influence (11.8 MPa), then using a tape yarn braided fabric ( 8.6 MPa), followed by using a fabric with a low initial pitch angle ( 7.2 $\mathrm{MPa}$ ). Meanwhile, in terms of deformation of the fabric, the angular reduction was mostly affected by the changing fabric

(i)

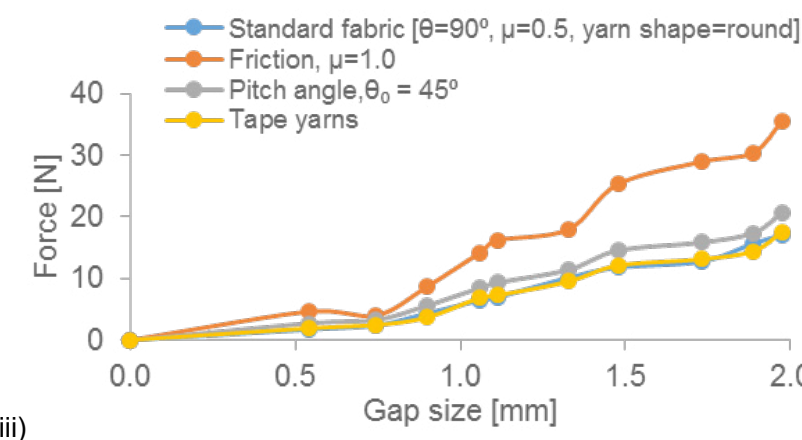

(iii)

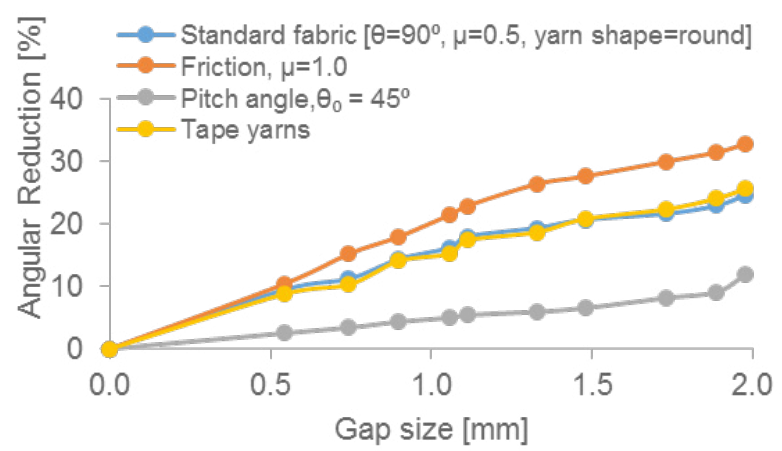

friction (32.8\%), followed by using a tape yarn braided fabric (25.6), and then using a fabric with a less initial pitch angle $(11.8 \%)$. The radial constriction of the fabric was mainly influenced by fabric friction (15.5\%), followed by using tape yarn braided fabric $(12.6 \%)$ and then using a fabric with a low initial pitch angle (6.3\%).

\subsubsection{Parameter analysis of repair potential of nitinol braided fabric}

In the analysis of parameters for the potential of using a nitinol braided fabric in tendon repair, it was noted that when there was an increase in fabric friction, the tendons will be displaced by $40.0 \mathrm{~mm}$; while at low fabric initial pitch angle, the tendons were displaced by $30.0 \mathrm{~mm}$, whereas with a tape yarn braided fabric, a displacement of $12.0 \mathrm{~mm}$ is required to form a gap of $2.0 \mathrm{~mm}$. The results for the evolution of gap between the tendons for different parameter analyzed are shown by the plots in Figure 11. In the analysis of the results for potential of using a nitinol braided fabric in tendon repair, as summarized

(ii)
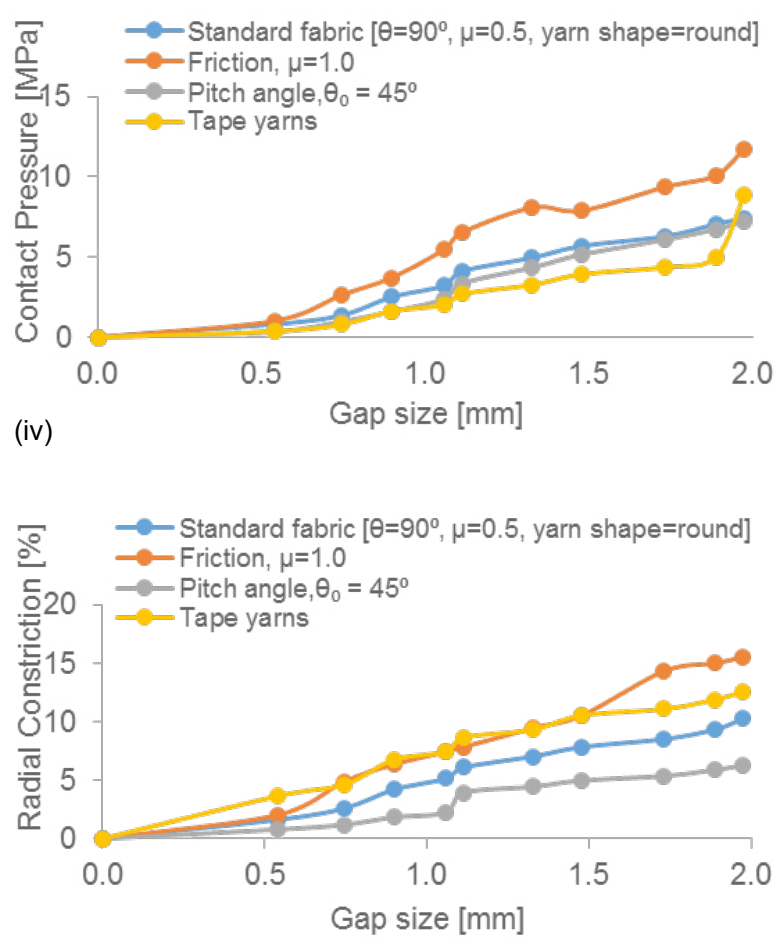

Figure 10: Analysis results for polyester braided fabric repair models at increased contact friction showing (i) force - gap size, (ii) contact pressure - gap, (iii) percentage angular reduction - gap and (iv) percentage radial constriction - gap.

Table 1: Analysis of parameter for polyester braided fabric tendon repair capabilities

\begin{tabular}{|c|c|c|c|c|c|}
\hline Parameter & d $(\mathrm{mm})$ & Load (N) & $\Delta \theta(\%)$ & $\Delta \mathbf{R}(\%)$ & CPRESS (MPa) \\
\hline Standard structure & 12.0 & 17.1 & 24.6 & 10.3 & 7.4 \\
\hline Increased friction & 22.0 & 35.6 & 32.8 & 15.5 & 11.8 \\
\hline Low initial pitch angle & 12.0 & 20.7 & 11.8 & 6.3 & 7.2 \\
\hline Tape yarns & 12.0 & 17.6 & 25.6 & 12.6 & 8.6 \\
\hline
\end{tabular}


in Table 2, when the parameters of the standard nitinol braided fabric were changed, it was noted that in terms of force in the repair required to form a $2 \mathrm{~mm}$ gap, changing the contact friction in the fabric will increase the force most $(83.3 \mathrm{~N})$. This was followed by using a fabric with a less initial pitch angle (73.0N); while using a tape yarn braided fabric would have less difference in the force $(18.7 \mathrm{~N})$.

In terms of contact pressure at the tendon-fabric interface, changing the friction in the fabric would also have the most effect (29.8MPa), followed by using a fabric with a low initial pitch angle (12.0MPa), and then using tape yarn braided fabric (11.6). In case of angular reduction, contact friction had the most influence $(32.9 \%)$, followed by using tape yarns $(17.6 \%)$, and then a fabric with a low initial angle (16.6\%). The radial constriction was mainly affected by using a fabric with contact friction $(21.6 \%)$, this was followed by tape yarn braided fabric $(8.2 \%)$, with low initial pitch angle fabric $(7.3 \%)$ having the least influence.

(i)

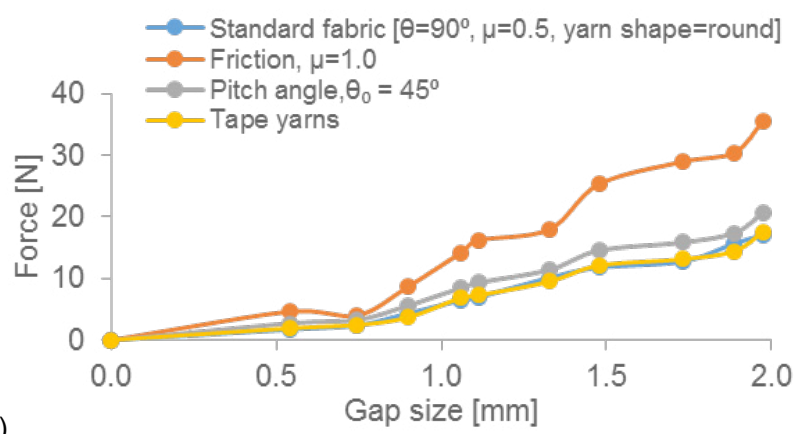

(iii)

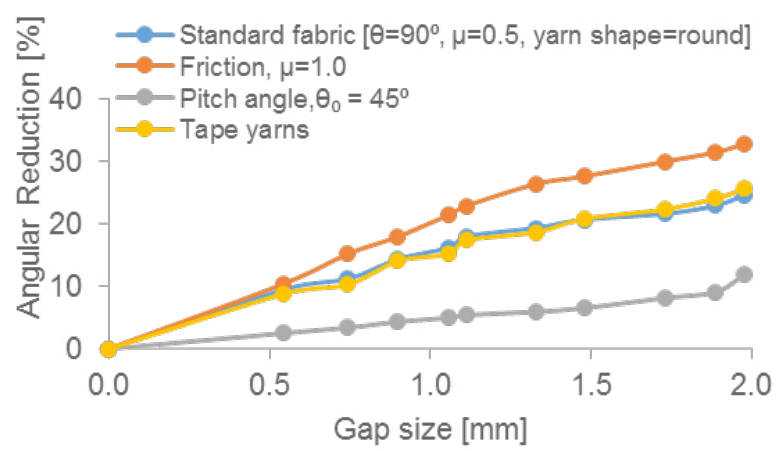

\section{Discussion}

The results for the potential of polyester and nitinol repair models indicate that an increase in contact friction will lead to a significant increase in the tension in the tendons and contact pressure in the repair process. This can be linked to increase in the rubbing effect at the yarn-yarn and tendon-braid interface, which would eventually cause an increase of abrasion in the repair process. It was also illustrated that using a braided fabric with smaller initial pitch angle would significantly increase the resistance to gap formation, as evidenced by the increase in the amount of force required to form the $2 \mathrm{~mm}$ gap, while only resulting in low deformation of the braided fabric. This implies that there will be less pressure on the tendons, as evidenced by the low change in the contact pressure on the tendons in both repair models, and could prove beneficial for the healing tendon. When a tape yarn was adopted for the braided fabrics, there was a significant change in the contact pressure in the repair models which can be attributed to the increase in contact surface of the

(ii)
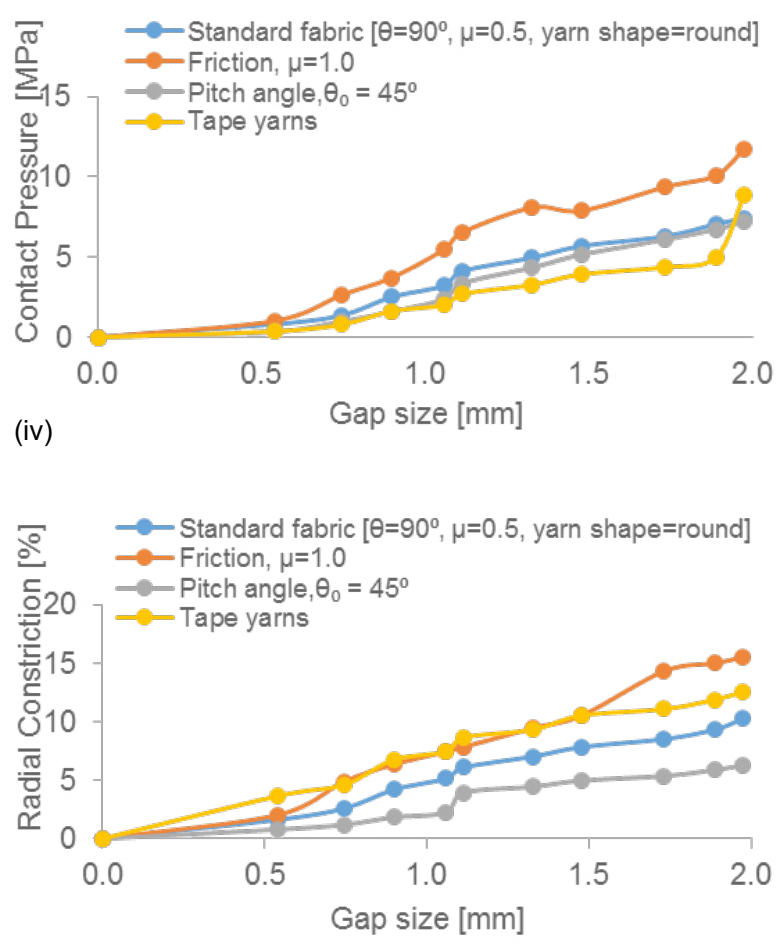

Figure 11: Analysis results for nitinol braided fabric repair models at increased contact friction showing (i) Force - gap size, (ii) stress - gap, (iii) percentage angular reduction - gap and (iv) percentage radial constriction - gap.

Table 2: Analysis of parameter for nitinol braided fabric tendon repair capabilities

\begin{tabular}{|c|c|c|c|c|c|}
\hline Parameter & $\mathrm{d}(\mathrm{mm})$ & Load (N) & $\Delta \theta(\%)$ & $\Delta \mathbf{R}(\%)$ & CPRESS (MPa) \\
\hline Standard fabric & 12.0 & 17.5 & 18.4 & 14.0 & 10.9 \\
\hline Low initial pitch angle & 30.0 & 73.0 & 16.6 & 7.3 & 12.0 \\
\hline Tape yarn braided fabric & 12.0 & 18.7 & 17.6 & 8.2 & 11.6 \\
\hline
\end{tabular}


tape yarns as compared to the normal round yarns. There was also evidence of high deformation of the braided fabric made of nitinol yarns, which could be attributed to the higher stiffness of the nitinol material. It was also established from the results that the tape yarns resulted in the least change for most parts of the repair models compared to a standard fabric; this implies that they could be better suited for tendon repair than the normal round yarns. This is because of the fact that the tape yarns have more surface area along the length of the braid fabric that would offer more support for the ruptured tendons at the repair site.

\section{Conclusions}

In this paper, the potential of a tubular braided fabric in repair of flexor tendons was studied using uniaxial tensile loading results from FEM and experimental test. In this regard, an FE model was developed in $A B A Q U S \circledR$ software based on numerical python script and validated with actual data from tensile loading tests on braided fabric repaired tendon models. The results from the FEM and experimental tests had an approximate agreement, which proves the validity of the FE model, and thus its application to simulate the evolution of gap size with tensile load of a braided fabric repaired tendon. The uniaxial tensile load recorded in the multi-braided fabric repair model was higher as compared to the regular-braided fabric repair model. This was attributed to the fact that a multi-braided fabric is inherently stiffer than a regular-braided fabric and would hence offer more stiffness during tendon repair. This was also evidenced by the deformation recorded for the multi-braided fabric model which was comparatively more than that in the regular-braided fabric repair model. The parameters of the regular braided fabric were changed by varying its material properties, contact friction, initial pitch angle, and yarn cross section variations were undertaken to investigate their impact on tendon repair process. The results illustrated changing the fabric parameters would significantly affect the gap formation, amount of force and deformation of the braided fabric during tendon repair. The results portrayed that increasing the contact friction coefficient of the braided fabrics had the most significant increase in the tension in the tendons and contact pressure in the repair process, than decreasing the initial pitch angle of fabrics or adopting tape yarns in the braided fabrics. The use of tape yarn braided fabrics resulted in the least change for most parts of the repair models and could be better suited for repair of tendons as compared to the normal round yarns. It can hence be concluded that these insights might prove helpful for future research/applications as they indicate what other types of braiding should be used.

\section{Acknowledgements}

The authors are grateful for the funding from the VLIR-UOS textile project.

\section{References}

[1] Drechsler K. (1999). 3-D Textile Reinforced Composites for the Transportation Industry. 3-D Textile Reinforcements in Composite Materials; 43-66.
[2] Tong, L., Adrian P.M., and Michael K.B. (2002). 3D Fiber Reinforced Polymer Composites; 137-146.

[3] Chellamani, K.P. and Indra Doraisamy. (2008). Recent advances in medical textiles. Asian Textile J. pp.49-55.

[4] Athanasios G P., Islam J., Alexandra Z., Stefanos K., Faruquz Z., Junaid M., and Noor B. (2011). New developments in the use of prostatic stents. Open Access Journal of Urology: 3.

[5] Chellamani, K.P., Sudharsan, J. and Sathish, J. (2013). Medical textiles using Braiding Technology. Journal of Academia and Industrial Research. 2(1), pp.21-26.

[6] Krasimira H., Eileen A., Matt D., and Christopher P. (2004). Mechanical Behavior of Circular Hybrid Braids under Tensile Loads Textile Research Journal 74(1) 20-26.

[7] Freitas, A.F., Araujo, M.D., Zu, W.W. and Fangueiro, R.M. (2010). Development of weft-knitted and braided polypropylene stents for arterial implant. The Journal of the Textile Institute. 101(12), 1027-1034.

[8] Ning, F., Potluri, P., Yu, W., and Hearle, J. (2016). Geometrical modeling of tubular braided structures using generalized rose curve. Textile Research Journal.

[9] Tuba A. (2012). 3D geometrical modelling of tubular Braids. Textile Research Journal. 82(5) 443-453.

[10] Nuutinen, J., Clerc, C., Reinikainen, R., and Törmälä, P. (2003). Mechanical properties and in vitro degradation of bioabsorbable self-expanding braided stents J. Biomater. Sci. Polymer Edn, Vol. 14, No. 3, pp. 255-266

[11] Irsale, S., and Adanur, S. (2006). Design and Characterization of Polymeric Stents. Journal of Industrial Textiles, 35(3):189.

[12] Rawson, S. D., Margetts, L., Wong, K. F., and Cartmell, S. H. (2015). Sutured tendon repair; a multi-scale finite element model. Biomech. Model. Mechanobiol. 14:123133.

[13] Chizari, M., Wang, B. and Snow, M. (2010). Effect of Suturing of a Tendon Graft in ACL Reconstruction. Engineering Letters, 17:3.

[14] Kubota, H., Aoki, M., Pruitt, D. L., and Manske P. R. (1996). Mechanical Properties of Various Circumferential Tendon Suture Techniques. J Hand Surg Eur Vol 21 (4) 474-480.

[15] Mathew, B., inventor; (2013) Jan. 10. Tendon repair device method. United States patent US 2013/0013065A1.

[16] Bower, A. (2009). Applied Mechanics of Solids. CRC Press.

[17] Moriya, T., Zhao, C., Yamashita, T., An, K., and Amadio, P. (2010). Effect of Core Suture Technique and Type on the Gliding Resistance during Cyclic Motion following Flexor Tendon Repair: A Cadaveric Study. J Orthop Res.; 28(11): 1475-1481.

[18] Silva, J., Zhao, C., An, K., Zobitz, M., Amadio, P. (2009), Gliding Resistance and Strength of Composite Sutures in Human Flexor Digitorum Profundus Tendon Repair: An In Vitro Biomechanical Study. J. Hand Surg. 34(A), pp. 87-92

[19] Tanaka, T. Amadio, P. C, Zhao, C, Zobitz, M. E., Yang, C, and An, K. N., (2004), Gliding Characteristics and Gap Formation for Locking and Grasping Tendon Repairs: A Biomechanical Study in a Human Cadaver Model," J. Hand Surg., 29A, pp. 6-14.

[20] $W u, Y$ and Tang, J. (2014). Recent developments in flexor tendon repair techniques and factors influencing strength of the tendon repair. J. Hand Surg., Vol 39E(1) 6-19. 
[21] Peterson J and Vegborn E. (2001). Development of a pre-knitting friction test method and study of friction and bending of yarns with high stiffness. Master of Science course in Textile Technology at the School of Textiles, University College of Bora's, Sweden, The University College of Bora's library database. http://bada.hb.se/ bitstream/2320/4525/1/BADA-joel-2008-9xjobb.pdf (accessed January 2016).

[22] Yoon, H. N. Sawyer, L. C. And Buckley A. (1984). Improved Comfort Polyester Part II: Mechanical and Surface Properties. Textile Research Journal vol. 54 no. 6 357-365

[23] Dassault Systèmes. Abaqus 6.14 User's Manual. Dassault Systèmes, (2014).

[24] Zhao, Shijia; Liu, Xiangyi (Cheryl); and Gu, Linxia, (2012). The Impact of Wire Stent Fabrication Technique on the Performance of Stent Placement. Mechanical \& Materials Engineering Faculty Publications. pp 61.

[25] Harte, A.-M., and Fleck, N. A. (2000). Deformation and Failure Mechanisms of Braided Composite Tubes in Compression and Torsion. Acta Materiala. 48 1259-1271.
[26] Yüksekkaya, M. E. (2001). Analysis of Elastic Deformation of Braided Tubular Structures for Medical Applications. Journal of Engineering Sciences.7(2): 277-285

[27] Goff, R.J. (1976). The Geometry of Tubular Braided Structures A Thesis Presented To The Faculty Of The Division Of Graduate Studies And Research. Master of Science in Textiles. Georgia Institute of Technology.

[28] Harte, A-M., and Norman A. (2000). On the mechanics of braided composites in tension. Fleck. Eur. J. Mech. A/ Solids Éditions scientifiques et médicales Elsevier SAS. 19 259-275.

[29] Viinikainen, A., Göransson, H., Ryhänen J. (2008). Primary Flexor Tendon Repair Techniques. Scandinavian Journal of Surgery 97: 333-340.

[30] Shah A., Rowlands, Megan, Au, A. (2015). Barbed Sutures and Tendon Repair-a Review. HAND 10:6-15. American Association for Hand Surgery.

[31] Zobitz, M., Zhao, C., Amadio, P., An, K. (2000). Comparison of mechanical properties of various suture repairtechniques in a partially lacerated tendon. J Biomech Eng. Dec 122(6) 604-7. 